\title{
PERIODIC BEURLING BOEHMIANS AND ANALYTIC BOEHMIANS *
}

\author{
V. KARUNAKARAN ${ }^{\dagger}$ AND R. ROOPKUMAR $\ddagger$
}

\begin{abstract}
We define Periodic Beurling Boehmians and analytic Boehmians. We also define boundary value of an analytic Boehmian as a periodic Beurling Boehmian and prove that each periodic Beurling Boehmian $x$ can be written as $x_{+}+\check{x}_{-}$where $x_{+}$and $x_{-}$are boundary values of certain analytic Boehmians.
\end{abstract}

1. Introduction. In [4], a space of periodic Boehmians containing the space of periodic distributions was introduced and a sufficient condition for a complex sequence to be the Fourier coefficients of a periodic Boehmian was given. A question regarding how a Boehmian could be associated to the boundary value of an analytic function, was raised. On the other hand, it is well known that (see for example [3]) every periodic distribution having all negative Fourier coefficients as zeros, can be written as the boundary value of a Bieberbach function.

In this paper we introduce a Boehmian space (called periodic Beurling Boehmians) which contains the set of all periodic Beurling distributions [2] and see how we can associate boundary values of analytic functions, to the elements of this Boehmian space. For this purpose we define another Boehmian space whose top space consists of analytic functions. We also define the concept of the boundary values of these Boehmians and prove that each periodic Beurling Boehmian can be expressed as a sum of boundary values of analytic Boehmians. Since periodic Beurling Boehmians are objects more general than periodic Beurling distributions, this representation is perhaps the best that one can expect in this context. Thereby answering partially the question posed in [4], in the most general set up.

We shall use the standard notations $\mathbf{C}, \mathbf{T}, U$ respectively for the finite complex plane, the unit circle and the open unit disc and as usual $\mathbf{N}, \mathbf{N}_{0}, \mathbf{Z}$ and $\mathbf{R}$ to denote the set of all natural numbers, non-negative integers, integers and reals.

In section 2 we recall the classical theory stating the required theorems with proper references. In section 3 the theory of periodic Beurling Boehmians is developed and in section 4 the theory of analytic Boehmians is developed and in section 5 we obtain results regarding boundary values of analytic functions associated with periodic Beurling distributions and establish our main result. Finally we give an example of a periodic Beurling Boehmian not representing any periodic Beurling distribution.

2. Classical theory. We denote by for $(k \in \mathbf{Z}), e_{k}$ the function

$$
e_{k}\left(e^{i t}\right)=e^{i k t}, \forall t \in[-\pi, \pi] \text {. }
$$

In this section we briefly recall the space of periodic Beurling distributions introduced in [2]. Let $\omega$ be a real valued function on $\mathbf{Z}$ satisfying the following properties:

( $\alpha) 0=\omega(0) \leq \omega(m+n) \leq \omega(m)+\omega(n), \forall m, n \in \mathbf{Z}$;

(ß) $\sum_{n \in \mathbf{Z}} \frac{\omega(n)}{1+n^{2}}<\infty$

\footnotetext{
*Received January 16, 2003; accepted for publication August 6, 2003.

${ }^{\dagger}$ School of Mathematics, Madurai Kamaraj University, Madurai, India (vkarun_mku@yahoo.co.in).

${ }^{\ddagger}$ School of Mathematics, Madurai Kamaraj University, Madurai, India. The work of this author is supported by a Senior Research Fellowship from CSIR, India.
} 
$(\gamma)$ there exist $a \in \mathbf{R}$ and $b>0$ such that $\omega(n) \geq a+b \log (1+|n|), \forall n \in \mathbf{Z}$;

$(\delta) \omega$ is concave down on $\mathbf{N}_{0}$ and is even.

Let $\mathcal{P}_{\omega}$ denote the space of all integrable complex valued functions $\theta$ on the unit circle $T$ with

$$
\|\theta\|_{\lambda}=\sum_{n \in \mathbf{Z}}|\hat{\theta}(n)| e^{\lambda \omega(n)}<\infty, \quad \forall \lambda>0 .
$$

with the Fréchet space topology given by the sequence of semi-norms $\|\cdot\|_{m}, m \in \mathbf{N}$. Here $\hat{\theta}(n)$ denotes the Fourier coefficient of $\theta$ defined by

$$
\hat{\theta}(n)=\int_{T} \theta e_{-n} d \sigma=\frac{1}{2 \pi} \int_{-\pi}^{\pi} \theta\left(e^{i t}\right) e^{-i n t} d t,
$$

where $d \sigma$ is the usual Haar measure [7, p.193] defined on $T$. The dual space $\mathcal{P}_{\omega}^{\prime}$ is called the space of Beurling distributions on $T$ which is algebraically isomorphic to the space of periodic Beurling distributions [2]. So throughout this paper we call $\mathcal{P}_{\omega}^{\prime}$ as the space of periodic Beurling distributions. In the article [2], it is remarked that if $\omega(n)=\log (1+|n|), \forall n \in \mathbf{Z}$ then $\mathcal{P}_{\omega}^{\prime}$ is same as the usual Schwartz periodic distributions. $\mathcal{P}_{\omega}$.

TheOREM 2.1. If $\theta \in \mathcal{P}_{\omega}$ then the Fourier series $\sum_{k \in \mathbf{Z}} \hat{\theta}(k) e_{k}$ converges to $\theta$ in

Proof. Let $\lambda>0$ be arbitrary. If $P_{N}=\sum_{-N}^{N} \hat{\theta}(k) e_{k}$ then

$$
\begin{aligned}
\left\|\theta-P_{N}\right\|_{\lambda} & =\sum_{k \in \mathbf{Z}}\left|\hat{\theta}(k)-\hat{P}_{N}(k)\right| e^{\lambda \omega(k)} \\
& =\sum_{|k| \geq N+1}|\hat{\theta}(k)| e^{\lambda \omega(k)} \rightarrow 0 \text { as } N \rightarrow \infty,
\end{aligned}
$$

being the tail end of the convergent series $\sum_{k \in \mathbf{Z}}|\hat{\theta}(k)| e^{\lambda \omega(k)}$.

Hence $P_{N} \rightarrow \theta$ as $N \rightarrow \infty$ in $\mathcal{P}_{\omega}$.

Definition 2.2. If $u \in \mathcal{P}_{\omega}^{\prime}$ then the Fourier coefficients $\hat{u}(k)$ of $u$ are defined by

$$
\hat{u}(k)=\left\langle u, e_{-k}\right\rangle, \forall k \in \mathbf{Z} .
$$

The proofs of the following theorems can be had from [2].

TheOREM 2.3. If $u \in \mathcal{P}_{\omega}^{\prime}$ then the Fourier series $\sum_{k \in \mathbf{Z}} \hat{u}(k) e_{k}$ converges to $u$ in $\mathcal{P}_{\omega}^{\prime}$.

TheOREM 2.4. If $u \in \mathcal{P}_{\omega}^{\prime}$ then there exist $C>0$ and $\lambda>0$ such that $|\hat{u}(k)| \leq$ $C e^{\lambda \omega(k)}, \forall k \in \mathbf{Z}$. Conversely, given a sequence of complex numbers $\left(a_{k}\right)_{k \in \mathbf{Z}}$ with $\left|a_{k}\right| \leq C e^{\lambda \omega(k)}, \forall k \in \mathbf{Z}$ for some $C>0$ and $\lambda>0$, there exists $u \in \mathcal{P}_{\omega}^{\prime}$ such that $\hat{u}(k)=a_{k}, \quad \forall k \in \mathbf{Z}$.

The above theorem characterizes the periodic Beurling distributions by a growth condition on their Fourier coefficients. 
Definition 2.5. If $u \in \mathcal{P}_{\omega}^{\prime}$ then we define $\check{u}$ by

$$
\langle\check{u}, \theta\rangle=\langle u, \check{\theta}\rangle, \quad \theta \in \mathcal{P}_{\omega} .
$$

Definition 2.6. Given $u \in \mathcal{P}_{\omega}^{\prime}$ and $\theta \in \mathcal{P}_{\omega}$ we define the convolution by

$$
(u * \theta)\left(e^{i x}\right)=\left\langle u, \tau_{x} \check{\theta}\right\rangle, \forall x \in[-\pi, \pi],
$$

where $\check{\theta}\left(e^{i t}\right)=\theta\left(e^{-i t}\right)$ and $\left(\tau_{x} \theta\right)\left(e^{i t}\right)=\theta\left(e^{i(t-x)}\right), \forall t \in[-\pi, \pi]$.

It is not difficult to see that this convolution is same as the one defined in [2].

Lemma 2.7. If $u \in \mathcal{P}_{\omega}^{\prime}$ and $\theta \in \mathcal{P}_{\omega}$ then $u * \theta \in \mathcal{P}_{\omega} \subset \mathcal{P}_{\omega}^{\prime}$ and

$$
(u * \theta \hat{)}(n)=\hat{u}(n) \cdot \hat{\theta}(n), \forall n \in \mathbf{Z} .
$$

In [3], it is proved that given a periodic distribution $u$ with all negative Fourier coefficients equal to zero, there exists an analytic function

$$
f(z)=\sum_{n=1}^{\infty} \hat{u}(n) z^{n}
$$

on the unit $\operatorname{disc} U=\{z \in \mathbf{C}:|z|<1\}$ such that the boundary value distribution

$$
\lim _{r \rightarrow 1^{-}}\left\langle f\left(r e^{i t}\right), \phi\left(e^{i t}\right)\right\rangle
$$

associated with $f(z)$ is the original distribution $u$.

3. Periodic Beurling Boehmians. A Boehmian space is a generalized function space consisting of equivalence classes of convolution quotients. For more detail we refer the reader to [6]. In this section we shall construct Beurling Boehmians and discuss some preliminary results. As in the context of Boehmians we take the additive semi-group $G$ as the space of the periodic Beurling testing function space $\mathcal{P}_{\omega}, S=G$ and the delta sequences as the collection $\Delta$ of all sequences $\left(\epsilon_{n}\right)$ from $\mathcal{P}_{\omega}$ satisfying the following usual properties:

(i) $\int_{T} \epsilon_{n} d \sigma=1, \forall n \in \mathbf{N}$;

(ii) $\int_{T}\left|\epsilon_{n}\right| d \sigma<M, \forall n \in \mathbf{N}$ for some $M>0$;

(iii) $s\left(\epsilon_{n}\right)=\sup \left\{|t|: t \in[-\pi, \pi], \epsilon_{n}\left(e^{i t}\right) \neq 0\right\} \rightarrow 0$ as $n \rightarrow \infty$.

We can easily prove the following lemma using Fubini's theorem, and the fact that $s\left(\epsilon_{n} * \phi_{n}\right) \leq s\left(\epsilon_{n}\right)+s\left(\phi_{n}\right)$.

LEMMA 3.1. If $\left(\epsilon_{n}\right),\left(\phi_{n}\right) \in \Delta$ then $\left(\epsilon_{n} * \phi_{n}\right) \in \Delta$.

Lemma 3.2. If $\theta \in \mathcal{P}_{\omega}$ and $\left(\epsilon_{n}\right) \in \Delta$ then $\theta * \epsilon_{n} \rightarrow \theta$ as $n \rightarrow \infty$ in $\mathcal{P}_{\omega}$.

Proof. First we show that $\left|\hat{\epsilon}_{n}(k)-1\right| \leq M s\left(\epsilon_{n}\right)|k| \forall k \in \mathbf{Z}$.

$$
\begin{aligned}
\left|\hat{\epsilon}_{n}(k)-1\right| & =\left|\int_{T}\left(\epsilon_{n} e_{-k}-\epsilon_{n}\right) d \sigma\right| \\
& \leq \frac{1}{2 \pi} \int_{-\pi}^{\pi}\left|\epsilon_{n}\left(e^{i t}\right)\left(e^{-i k t}-1\right)\right| d t \\
& \leq \frac{1}{2 \pi} \int_{-\pi}^{\pi}\left|\epsilon_{n}\left(e^{i t}\right)\right| \cdot|k t| d t \quad \text { (By mean-value theorem) } \\
& \leq M s\left(\epsilon_{n}\right)|k| .
\end{aligned}
$$


Next consider for $\lambda>0$,

$$
\begin{aligned}
\left\|\theta * \epsilon_{n}-\theta\right\|_{\lambda}= & \sum_{k \in \mathbf{Z}} \mid\left(\theta * \epsilon_{n} \hat{)}(k)-\hat{\theta}(k) \mid e^{\lambda \omega(k)}\right. \\
= & \left.\sum_{k \in \mathbf{Z}}|\hat{\theta}(k)| \cdot\left|\hat{\epsilon}_{n}(k)-1\right| e^{\lambda \omega(k)} \quad \text { (By Lemma 2.7 }\right) \\
\leq & M s\left(\epsilon_{n}\right)\|\theta\|_{\mu} \sum_{k \in \mathbf{Z}}|k| e^{-(\mu-\lambda) \omega(k)} \\
& \left(\text { where } \mu \geq \frac{3}{b}+\lambda, \text { with 'b' as in property }(\gamma) \text { of } \omega .\right) \\
\leq & M s\left(\epsilon_{n}\right)\|\theta\|_{\mu} e^{-a(\mu-\lambda)} \sum_{k \in \mathbf{Z}} \frac{|k|}{(1+|k|)^{(\mu-\lambda) b} \quad(B y(\gamma))} \\
\leq & M^{\prime} s\left(\epsilon_{n}\right) \rightarrow 0 \text { as } n \rightarrow \infty \text { for some } 0<M^{\prime}<\infty .
\end{aligned}
$$

Lemma 3.3. If $\theta_{n} \rightarrow \theta$ as $n \rightarrow \infty$ in $\mathcal{P}_{\omega}$ and $\eta \in \mathcal{P}_{\omega}$, then $\theta_{n} * \eta \rightarrow \theta * \eta$ as $n \rightarrow \infty$ in $\mathcal{P}_{\omega}$.

Proof. Let $\lambda>0$ be arbitrary. Using Lemma 2.7 we get

Hence the lemma.

$$
\begin{aligned}
\left\|\theta_{n} * \eta-\theta * \eta\right\|_{\lambda} & =\sum_{k \in \mathbf{Z}}\left|\hat{\theta}_{n}(k) \cdot \hat{\eta}(k)-\hat{\theta}(k) \cdot \hat{\eta}(k)\right| e^{\lambda \omega(k)} \\
& \leq\|\eta\|_{1} \sum_{k \in \mathbf{Z}}\left|\hat{\theta}_{n}(k)-\hat{\theta}(k)\right| e^{\lambda \omega(k)} \\
& =\|\eta\|_{1}\left\|\theta_{n}-\theta\right\|_{\lambda} \rightarrow 0 \text { as } n \rightarrow \infty .
\end{aligned}
$$

Lemma 3.4. If $\theta_{n} \rightarrow \theta$ as $n \rightarrow \infty$ in $\mathcal{P}_{\omega}$ and $\left(\epsilon_{n}\right) \in \Delta$ then $\theta_{n} * \epsilon_{n} \rightarrow \theta$ as $n \rightarrow \infty$ in $\mathcal{P}_{\omega}$.

Proof. We have $\left\|\theta_{n} * \epsilon_{n}-\theta\right\|_{\lambda} \leq\left\|\theta_{n} * \epsilon_{n}-\theta * \epsilon_{n}\right\|_{\lambda}+\left\|\theta * \epsilon_{n}-\theta\right\|_{\lambda}$.

By Lemma 3.2, the second term tends to zero as $n \rightarrow \infty$. Consider the first term

$$
\begin{aligned}
\left\|\theta_{n} * \epsilon_{n}-\theta * \epsilon_{n}\right\|_{\lambda} & =\sum_{k \in \mathbf{Z}}\left|\hat{\theta}_{n}(k)-\hat{\theta}(k)\right| \cdot\left|\hat{\epsilon}_{n}(k)\right| e^{\lambda \omega(k)} \\
& \leq M\left\|\theta_{n}-\theta\right\|_{\lambda} . \quad\left(\text { using property }(i i) \text { of }\left(\epsilon_{n}\right) .\right)
\end{aligned}
$$

This completes the proof of the lemma.

Now we can construct the space of Boehmians as follows: A pair of sequences $\left(\left(\theta_{n}\right),\left(\epsilon_{n}\right)\right)$ is said to be a quotient if

$$
\theta_{n} * \epsilon_{m}=\theta_{m} * \epsilon_{n}, \forall m, n \in \mathbf{N} .
$$

The collection of all quotients is denoted by $\mathcal{A}$ and each quotient is denoted by $\theta_{n} / \epsilon_{n}$. Now we define an equivalence relation $\sim$ on $\mathcal{A}$ by $\theta_{n} / \epsilon_{n} \sim \eta_{n} / \varphi_{n}$ if $\theta_{n} * \varphi_{m}=\eta_{m} * \epsilon_{n}$ for all $m, n \in \mathbf{N}$. The collection of equivalence classes is denoted by $\mathcal{B}_{\omega}$ and each equivalence class containing $\theta_{n} / \epsilon_{n}$ is denoted by $\left[\theta_{n} / \epsilon_{n}\right]$, and is called a periodic Beurling Boehmian. It is easy to verify that if $\omega(n)=\log (1+|n|)$ then $\mathcal{B}_{\omega}$ is same as the space of periodic Boehmians defined in $[4,5]$.

The algebraic operations such as addition, scalar multiplication and the convolution of a Boehmian and periodic Beurling testing function are defined by

(a) $\left[\theta_{n} / \epsilon_{n}\right]+\left[\eta_{n} / \varphi_{n}\right]=\left[\left(\theta_{n} * \varphi_{n}+\eta_{n} * \epsilon_{n}\right) /\left(\phi_{n} * \epsilon_{n}\right)\right]$.

(b) $\alpha\left[\theta_{n} / \epsilon_{n}\right]=\left[\alpha \theta_{n} / \epsilon_{n}\right]$.

(c) $\left[\theta_{n} / \epsilon_{n}\right] * \eta=\left[\left(\theta_{n} * \eta\right) / \epsilon_{n}\right]$.

Each $u \in \mathcal{P}_{\omega}^{\prime}$ is identified as a Boehmian whose representative is $\left(u * \epsilon_{n}\right) / \epsilon_{n}$ where $\left(\epsilon_{n}\right) \in \Delta$ is arbitrary.

Definition 3.5. We say that a sequence $\left(x_{n}\right)$ of Boehmians is $\delta$-convergent to a Boehmian $x$ if there exists $\left(\epsilon_{m}\right) \in \Delta$ such that

$$
x_{n} * \epsilon_{m}, x * \epsilon_{m} \in \mathcal{P}_{\omega}, \quad \forall m, n \in \mathbf{N}
$$


and for each $m \in \mathbf{N}$,

$$
x_{n} * \epsilon_{m} \rightarrow x * \epsilon_{m} \text { as } n \rightarrow \infty
$$

We write $x_{n} \stackrel{\delta}{\rightarrow} x$ as $n \rightarrow \infty$.

We denote this limit $x$ as $\delta$ - $\lim _{n \rightarrow \infty} x_{n} . \quad \delta$-convergence normally does not induce a topological convergence, however, it does possess many desired properties. For example the limit of a convergent sequence is unique; a subsequence of a convergent sequence is convergent to the same limit; the sum of two convergent sequence is convergent to the sum of their limits.

LEMma 3.6. (See [6]) $x_{n} \stackrel{\delta}{\rightarrow} x$ as $n \rightarrow \infty$ if and only if there exist representatives $\theta_{n, k} / \epsilon_{k}, \quad \theta_{k} / \epsilon_{k}$ of $x_{n}$ and $x$ respectively such that for each $k \in \mathbf{N}$, $\theta_{n, k} \rightarrow \theta_{k}$ as $n \rightarrow \infty$ in $\mathcal{P}_{\omega}$.

Definition 3.7. If $x=\left[\theta_{n} / \epsilon_{n}\right] \in \mathcal{B}_{\omega}$ then define $\check{x}=\left[\check{\theta}_{n} / \check{\epsilon}_{n}\right]$.

We can prove that the $\check{x}$ for each $x \in \mathcal{B}_{\omega}$ well defined, by using the fact that $\left(\phi * \psi \check{\psi}=\check{\phi} * \check{\psi}, \forall \phi, \psi \in \mathcal{P}_{\omega}\right.$.

The proofs of the following two theorems are straight forward and we prefer to omit the details.

TheOREM 3.8. If $x, y \in \mathcal{B}_{\omega}$ then $(i)(x+y)=\check{x}+\check{y}$; (ii) $(\alpha x)=\alpha \check{x}$; (iii) $\check{\check{x}}=x$.

TheOREM 3.9. The map $x \mapsto \check{x}$ is a homeomorphism of $\mathcal{B}_{\omega}$ onto itself.

Definition 3.10. Given $x=\left[\theta_{n} / \epsilon_{n}\right] \in \mathcal{B}_{\omega}$ and $k \in \mathbf{N}$ define the Fourier coefficient of $x$ by $\hat{x}(k)=\frac{\hat{\theta}_{n}(k)}{\hat{\epsilon}_{n}(k)}$ if $\hat{\epsilon}_{n}(k) \neq 0$.

We note that given $k \in \mathbf{N}$, there exists $n \in \mathbf{N}$ such that $\hat{\epsilon}_{n}(k) \neq 0$ because $\hat{\epsilon}_{n}(k) \rightarrow 1$ as $n \rightarrow \infty$. If $\epsilon_{n}(k) \neq 0 \neq \epsilon_{m}(k)$, the equality $\theta_{n} * \epsilon_{m}=\theta_{m} * \epsilon_{n}$ implies that $\frac{\hat{\theta}_{n}(k)}{\hat{\epsilon}_{n}(k)}=\frac{\hat{\theta}_{m}(k)}{\hat{\epsilon}_{m}(k)}$.

Moreover if $x=\left[\theta_{n} / \epsilon_{n}\right]=\left[\eta_{n} / \varphi_{n}\right]$ and $\hat{\epsilon}_{n}(k) \neq 0 \neq \hat{\varphi}_{m}(k)$ then we get $\frac{\hat{\theta}_{n}(k)}{\hat{\epsilon}_{n}(k)}=$ $\frac{\hat{\eta}_{m}(k)}{\hat{\varphi}_{m}(k)}$ by using the equality $\theta_{n} * \varphi_{m}=\eta_{m} * \epsilon_{n}$. Thus the definition is independent of the choice of the representative and hence $\hat{x}(k)$ is well defined.

Theorem 3.11. If $x=\left[\theta_{n} / \epsilon_{n}\right] \in \mathcal{B}_{\omega}$, then the Fourier series $\sum_{k \in \mathbf{Z}} \hat{x}(k) e_{k}$ $\delta$-converges to $x$.

Proof. To prove this theorem we shall show that for each $j \in \mathbf{N}$,

$$
\sum_{k=-N}^{N} \hat{x}(k) e_{k} * \epsilon_{j} \rightarrow \theta_{j} \text { as } N \rightarrow \infty \text { in } \mathcal{P}_{\omega} .
$$

$$
\begin{aligned}
\text { Now }\left(\sum_{k=-N}^{N} \hat{x}(k) e_{k} * \epsilon_{j}\right)\left(e^{i s}\right) & =\sum_{k=-N}^{N} \hat{x}(k) \frac{1}{2 \pi} \int_{-\pi}^{\pi} e^{i k(s-t)} \epsilon_{j}\left(e^{i t}\right) d t \\
& =\sum_{k=-N}^{N} \hat{x}(k) e^{i k s} \frac{1}{2 \pi} \int_{-\pi}^{\pi} e^{-i k t} \epsilon_{j}\left(e^{i t}\right) d t \\
& =\sum_{k=-N}^{N} \hat{x}(k) e^{i k s} \hat{\epsilon}_{j}(k) .
\end{aligned}
$$


If $\hat{\epsilon}_{j}(k)=0$ then, for this $k \in \mathbf{Z}$, we can find $l \in \mathbf{N}$ such that $\hat{\epsilon}_{l}(k) \neq 0$. Now $\theta_{l} * \epsilon_{j}=\theta_{j} * \epsilon_{l}$ implies $\hat{\theta}_{l}(k) \cdot \hat{\epsilon}_{j}(k)=\hat{\theta}_{j}(k) \cdot \hat{\epsilon}_{l}(k)$. Therefore it follows that $\hat{\theta}_{j}(k)=0$. If $\hat{\epsilon}_{j}(k) \neq 0$, then by definition of $\hat{x}(k)$, we get $\hat{x}(k) \cdot \hat{\epsilon}_{j}(k)=\hat{\theta}_{j}(k)$. Therefore $\sum_{k=-N}^{N} \hat{x}(k) e^{i k s} \hat{\epsilon}_{j}(k)=\sum_{k=-N}^{N} \hat{\theta}_{j}(k) e^{i k s}$ which converges to $\theta_{j}$ in $\mathcal{P}_{\omega}$ by Theorem 2.1. Hence the result.

4. Analytic Boehmians. In this section we define a Boehmian space whose top space $G$ consists of analytic functions in the open unit disc $U$ of $\mathbf{C}$, called analytic Boehmians. To construct this space we take $G=S=\left\{f(z)=\sum_{k=0}^{\infty} a_{k} z^{k}:\left|a_{k}\right| \leq\right.$ $C e^{-\lambda \omega(k)} \forall k \in \mathbf{N}_{0}$, and for each $\left.\lambda>0\right\}$. Here the constant $C$ may depend on $\lambda$. Note that $G \subset H(U)$ ( the collection of all holomorphic functions on $U$ ). We also take the multiplication in $G$ as the Hadamard product defined by

$$
\sum_{k=0}^{\infty} a_{k} z^{k} \star \sum_{k=0}^{\infty} b_{k} z^{k}=\sum_{k=0}^{\infty}\left(a_{k} \cdot b_{k}\right) z^{k} .
$$

LEMMA 4.1. If $f, g \in G$ then $f \star g \in G$.

Proof. Let $f(z)=\sum_{k=0}^{\infty} a_{k} z^{k}, \quad g(z)=b_{k} z^{k} \in G$. Now $(f \star g)(z)=\sum_{k=0}^{\infty}\left(a_{k} \cdot b_{k}\right) z^{k}$. Consider for $\lambda>0,\left|a_{k} \cdot b_{k}\right| e^{\lambda \omega(k)}=\left|a_{k}\right| e^{\frac{\lambda}{2} \omega(k)} \cdot\left|b_{k}\right| e^{\frac{\lambda}{2} \omega(k)} \leq C_{1} \cdot C_{2}$ where $C_{1}, C_{2}$ are such that $\left|a_{k}\right| \leq C_{1} e^{-\frac{\lambda}{2} \omega(k)}, \quad\left|b_{k}\right| \leq C_{2} e^{-\frac{\lambda}{2} \omega(k)}$. Therefore $f \star g \in G$. Hence the lemma follows.

The proof of the following lemma is obvious.

Lemma 4.2. If $f, g, h \in G$ and $\alpha \in \mathbf{C}$, then

(a) $f \star g=g \star f$.

(b) $(f \star g) \star h=f \star(g \star h)$.

(c) $(f+g) \star h=(f \star g)+(f \star h)$.

(d) $\alpha(f \star g)=(\alpha f) \star g$.

We define semi-norms on $G$ by $P_{\lambda}(f)=\sup _{k \geq 0}\left|a_{k}\right| e^{\lambda \omega(k)}$. It is easy to verify that $\left\{P_{\lambda}(\cdot): \lambda>0\right\}$ is an increasing family of semi-norms and hence the topology induced by this family of semi-norms is the same as that defined by $\left\{P_{m}(\cdot): m \in \mathbf{N}\right\}$. Therefore this space becomes a metrizable topological vector space.

Now we prove that this space is complete.

THEOREM 4.3. G is a complete metric space.

Proof. Let $\left(f_{n}\right)$ be a Cauchy sequence in $G$. Then for each $\lambda>0$, given $r>0$, there exists $N(\lambda) \in \mathbf{N}$ such that $P_{\lambda}\left(f_{n}-f_{m}\right)<r, \quad \forall n, m \geq N(\lambda)$. If $f_{n}(z)=$ $\sum_{k=0}^{\infty} a_{n, k} z^{k}$ then for all $k \in \mathbf{N}_{0}$, we have

$$
\left|a_{n, k}-a_{m, k}\right| \leq P_{\lambda}\left(f_{n}-f_{m}\right)<r, \quad \forall n, m \geq N(\lambda)
$$

and hence $a_{n, k}$ converges to $a_{k}$ uniformly in $k \in \mathbf{N}_{0}$. Hence we get that $\sup \left|a_{k}-a_{m, k}\right| e^{\lambda \omega(k)} \leq r, \forall m \geq N(\lambda)$. 
To prove $f \in G$ we consider (for each $\mu>0$ )

$$
\begin{aligned}
\sup _{k \geq 0}\left|a_{k}\right| e^{\mu \omega(k)} & \leq \sup _{k \geq 0}\left|a_{k}-a_{N(\mu), k}\right| e^{\mu \omega(k))}+\sup _{k \geq 0}\left|a_{N(\mu), k}\right| e^{\mu \omega(k)} \\
& \leq r+P_{\mu}\left(f_{N(\mu)}\right)<+\infty
\end{aligned}
$$

Therefore $f \in G$. By (I) we get that $f_{n} \rightarrow f$ as $n \rightarrow \infty$ in $G$. Hence the lemma.

Now we define delta sequences.

Definition 4.4. A sequence $\left(d_{n}\right)$ in $G$ is said to be a delta sequence if $d_{n}(z)=$ $\sum_{k=0}^{\infty} d_{n, k} z^{k}$ and

1. There exists $M>0$ such that $\left|d_{n, k}\right| \leq M, \forall n \in \mathbf{N}, \forall k \in \mathbf{N}_{0}$.

2. There exists a non-negative sequence $\left(\alpha_{n}\right)$ converging to 0 , such that

$$
\left|d_{n, k}-1\right| \leq \alpha_{n}|k|, \forall k \in \mathbf{N}_{0} .
$$

Lemma 4.5. If $\left(d_{n}\right)$ and $\left(e_{n}\right)$ are delta sequences, then $\left(d_{n} \star e_{n}\right)$ is also.

$$
\begin{gathered}
\text { Proof. Put } d_{n}(z)=\sum_{k=0}^{\infty} d_{n, k} z^{k}, \quad e_{n}(z)=\sum_{k=0}^{\infty} e_{n, k} z^{k} ; \text { then } \\
\left(d_{n} \star e_{n}\right)(z)=\sum_{k=0}^{\infty}\left(d_{n, k} \cdot e_{n, k}\right) z^{k} .
\end{gathered}
$$

Let $M_{1}, M_{2}$ be such that $\left|d_{n, k}\right| \leq M_{1}, \quad\left|e_{n, k}\right| \leq M_{2} \forall n \in \mathbf{N}, k \in \mathbf{N}_{0}$ and $\left(\alpha_{n}\right),\left(\beta_{n}\right)$ satisfy the property $(2)$ of delta sequence, corresponding to $\left(d_{n}\right),\left(e_{n}\right)$ respectively. Now $\left|d_{n, k} \cdot e_{n, k}\right| \leq M_{1} \cdot M_{2} \forall n \in \mathbf{N}, k \in \mathbf{N}_{0}$, and

$$
\left|d_{n, k} \cdot e_{n, k}-1\right| \leq\left|d_{n, k}-1\right| \cdot\left|e_{n, k}-1\right|+\left|d_{n, k}-1\right|+\left|e_{n, k}-1\right|
$$

Hence the lemma.

$$
\leq\left(M_{1}+1\right) \beta_{n}|k|+\alpha_{n}|k|+\beta_{n}|k| \text {. }
$$

LEMma 4.6. If $f_{n} \rightarrow f$ as $n \rightarrow \infty$ in $G$ and $g \in G$ then $f_{n} \star g \rightarrow f \star g$ as $n \rightarrow \infty$.

Proof. Let $\lambda>0$ and $f_{n}(z)=\sum_{k=0}^{\infty} a_{n, k} z^{k}, \quad f(z)=\sum_{k=0}^{\infty} a_{k} z^{k}, \quad g(z)=\sum_{k=0}^{\infty} b_{k} z^{k}$. Now the following inequalities complete the proof of this lemma

$$
\begin{aligned}
P_{\lambda}\left(f_{n} \star g-f \star g\right) & =\sup _{k \geq 0}\left|\left(a_{n, k}-a_{k}\right) \cdot b_{k}\right| e^{\lambda \omega(k)} \\
& \leq P_{1}(g) P_{\lambda}\left(f_{n}-f\right) .
\end{aligned}
$$

$G$.

LEMMA 4.7. If $f \in G$ and $\left(d_{n}\right)$ is a delta sequence then $f \star d_{n} \rightarrow f$ as $n \rightarrow \infty$ in

Proof. For $\lambda>0$, consider

$$
\begin{aligned}
P_{\lambda}\left(f \star d_{n}-f\right) & =\sup _{k \geq 0}\left|a_{k} \cdot d_{n, k}-a_{k}\right| e^{\lambda \omega(k)} \\
& \leq \alpha_{n} \sup _{k \geq 0}\left|a_{k}\right| \cdot|k| e^{\lambda \omega(k)} \quad\left(\text { by property }(2) \text { of }\left(d_{n}\right)\right) \\
& =\alpha_{n} P_{\mu}(f) \sup _{k \geq 0} e^{-\mu \omega(k)}|k| e^{\lambda \omega(k)} \quad\left(\text { where } \mu \geq \frac{2}{b}+\lambda\right) \\
& =\alpha_{n} P_{\mu}(f) e^{-a(\mu-\lambda)} \sup _{k \geq 0} \frac{|k|}{(1+|k|)^{(\mu-\lambda) b}} \\
& \leq \alpha_{n} P_{\mu}(f) e^{-a(\mu-\lambda)} \stackrel{\rightarrow}{\rightarrow} \text { as } n \rightarrow \infty .
\end{aligned}
$$

Therefore the lemma follows. 
LEMMA 4.8. If $f_{n} \rightarrow f$ as $n \rightarrow \infty$ in $G$ and $d_{n}$ is a delta sequence then $f_{n} \star d_{n} \rightarrow f$ as $n \rightarrow \infty$ in $G$.

Proof. Let $f_{n}(z)=\sum_{k=0}^{\infty} a_{n, k} z_{k}, \quad f(z)=\sum_{k=0}^{\infty} a_{k} z_{k}, \quad d_{n}(z)=\sum_{k=0}^{\infty} d_{n, k} z^{k}$. For $\lambda>0$, we have $P_{\lambda}\left(f_{n} \star d_{n}-f\right) \leq P_{\lambda}\left(f_{n} \star d_{n}-f \star d_{n}\right)+P_{\lambda}\left(f \star d_{n}-f\right)$ and the second term of the above inequality tends to zero as $n \rightarrow \infty$, by Lemma 4.7. Now consider

$$
\begin{aligned}
P_{\lambda}\left(f_{n} \star d_{n}-f \star d_{n}\right) & =\sup _{k \geq 0}\left|a_{n, k}-a_{k}\right| \cdot\left|d_{n, k}\right| e^{\lambda \omega(k)} \\
& \leq M \sup _{k \geq 0}\left|a_{n, k}-a_{k}\right| e^{\lambda \omega(k)} \rightarrow 0 \text { as } n \rightarrow \infty
\end{aligned}
$$

Now we can define the space of analytic Boehmians in the usual way.

If $F(z)=\sum_{k=0}^{\infty} a_{k} z^{k}$ with $\left|a_{k}\right| \leq C e^{\lambda \omega(k)}, k \in \mathbf{N}_{0}$, for some positive real numbers $C$ and $\lambda$, then we can identify $F$ in $\mathcal{B}_{A}$ by the Boehmian whose representative is $\left(F \star d_{n}\right) / d_{n}$ where $\left(d_{n}\right)$ is any delta sequence.

It is an easy exercise to verify that the above identification is well defined and is one-to-one.

\section{Boundary value theory.}

LEMMA 5.1. Let $\theta \in \mathcal{P}_{\omega}$. If $f_{+}(z)=\sum_{k=0}^{\infty} \hat{\theta}(k) z^{k}, f_{-}(z)=\sum_{k=1}^{\infty} \hat{\theta}(-k) z^{k}$ then $f_{+}, f_{-} \in G$ and $\theta=\lim _{r \rightarrow 1^{-}} f_{+}\left(r e^{i t}\right)+\left(\lim _{r \rightarrow 1^{-}} f_{-}\left(r e^{i t}\right)\right)$ (where these limits are taken in $\left.\mathcal{P}_{\omega}\right)$.

Proof. Define $f_{+}(z)=\sum_{k=0}^{\infty} \hat{\theta}(k) z^{k}$. Since for each $\lambda>0,|\hat{\theta}(k)| \leq\|\theta\|_{\lambda} e^{-\lambda \omega(k)}$, $\forall k \in Z$ we get $f_{+} \in G$. Now define $\theta_{+}\left(e^{i t}\right)=\sum_{k=0}^{\infty} \hat{\theta}(k) e^{i k t}$. First we show that $\theta_{+} \in \mathcal{P}_{\omega}$. Now for $\lambda>\frac{2}{b}$, where $b$ is as in the property $(\gamma)$ of $\omega$,

$$
\begin{aligned}
\sum_{k=0}^{\infty}\left|\hat{\theta}(k) e^{i k t}\right| & \leq \sum_{k=0}^{\infty}\|\theta\|_{\lambda} e^{-\lambda \omega(k)} \\
& \leq\|\theta\|_{\lambda} e^{-a \lambda} \sum_{k=0}^{\infty} \frac{1}{(1+|k|)^{b \lambda}}<\infty . \quad \text { (By the property }(\gamma) \text {.) }
\end{aligned}
$$

Therefore the above series converges uniformly to $\theta_{+}$and hence $\hat{\theta}_{+}(k)=\left\{\begin{array}{lll}\hat{\theta}(k) & \text { if } & k \in \mathbf{N}_{0} \\ 0 & \text { if } & k \notin \mathbf{N}_{0}\end{array}\right.$

This shows that $\theta_{+} \in \mathcal{P}_{\omega}$.

Now for $r \in(0,1)$, if $g_{r}\left(e^{i t}\right)=f_{+}\left(r e^{i t}\right)$ then, for $\lambda>0$,

$$
\begin{aligned}
\left\|\theta_{+}-g_{r}\right\|_{\lambda} & =\sum_{k \geq 1}\left|\left(1-r^{k}\right) \cdot \hat{\theta}(k)\right| e^{\lambda \omega(k)} \\
& \leq(1-r) \sum_{k \geq 1} k \cdot|\hat{\theta}(k)| e^{\lambda \omega(k)} \\
& \leq(1-r)\|\theta\|_{\mu} e^{-a(\mu-\lambda)} \sum_{k \geq 1} \frac{k}{(1+k)^{b(\mu-\lambda)}} \quad \text { for some } \mu>\frac{3}{b}+\lambda \\
& \leq C(1-r) \rightarrow 0 \text { as } r \rightarrow 1^{-} \quad(\text { for some } 0<C<\infty)
\end{aligned}
$$

By a similar argument we can show that if $f_{-}(z)=\sum_{k=1}^{\infty} \hat{\theta}(-k) z^{k}$ then $f_{-} \in G$, 
$\lim _{r \rightarrow 1^{-}} f_{-}\left(r e^{i t}\right)=\theta_{-}\left(e^{i t}\right)$ in $\mathcal{P}_{\omega}$, where $\theta_{-}\left(e^{i t}\right)=\sum_{k=1}^{\infty} \hat{\theta}(-k) e^{i k t}$ and $\hat{\theta}_{-}(k)=\left\{\begin{array}{lll}\hat{\theta}(-k) & \text { if } & k \in \mathbf{N} \\ 0 & \text { if } & k \notin \mathbf{N} .\end{array}\right.$

Note that for each $k \in \mathbf{Z},\left(\theta_{+}+\check{\theta}_{-}\right) \hat{(}(k)=\hat{\theta}_{+}(k)+\hat{\theta}_{-}(-k)=\hat{\theta}(k)$. Hence by Theorem 2.1, we get $\theta=\theta_{+}+\check{\theta}_{-}$. Hence the lemma follows.

Lemma 5.2. Let $u \in \mathcal{P}_{\omega}^{\prime}$. If $F_{+}(z)=\sum_{k=0}^{\infty} \hat{u}(k) z^{k}, F_{-}(z)=\sum_{k=1}^{\infty} \hat{u}(-k) z^{k}$ then $F_{+}, F_{-} \in G$ and $u=\lim _{r \rightarrow 1^{-}} F_{+}\left(r e^{i t}\right)+\left(\lim _{r \rightarrow 1^{-}} F_{-}\left(r e^{i t}\right)\right)$ (where these limits are taken in $\left.\mathcal{P}_{\omega}^{\prime}\right)$.

Proof. By Theorem 2.4, we have $|\hat{u}(k)| \leq C e^{\lambda \omega(k)}, \forall k \in \mathbf{Z}$ for some $C>0, \lambda>0$. By a remark mentioned in [5], we get $\limsup _{k \rightarrow \infty}|\hat{u}(k)|^{\frac{1}{k}} \leq 1$.

We define $F_{+}(z)=\sum_{k=0}^{\infty} \hat{u}(k) z^{k}$, then $F_{+}$is analytic on $U$. First we show that $\lim _{r \rightarrow 1^{-}} F_{+}\left(r e^{i t}\right)$ exists in $\mathcal{P}_{\omega}^{\prime}$. Let $\theta \in \mathcal{P}_{\omega}$,

$$
\begin{aligned}
\left\langle F_{+}\left(r e^{i t}\right), \theta\left(e^{i t}\right)\right\rangle & =\frac{1}{2 \pi} \int_{-\pi}^{\pi} F_{+}\left(r e^{i t}\right) \theta\left(e^{i t}\right) d t \\
& =\frac{1}{2 \pi} \int_{-\pi}^{\pi} \sum_{k=0}^{\infty} \hat{u}(k) r^{k} e^{i k t} \cdot \theta\left(e^{i t}\right) d t \\
& =\sum_{k=0}^{\infty} \hat{u}(k) r^{k} \frac{1}{2 \pi} \int_{-\pi}^{\pi} \theta\left(e^{i t}\right) e^{i k t} d t \\
& =\sum_{k=0}^{\infty} \hat{u}(k) r^{k} \hat{\theta}(-k) . \\
\left|\sum_{k=0}^{\infty} \hat{u}(k) \cdot \hat{\theta}(-k)\right| \leq & \sum_{k=0}^{\infty}|\hat{u}(k) \cdot \hat{\theta}(-k)| \\
\leq & C\|\theta\|_{\mu} \sum_{k=0}^{\infty} e^{-(\mu-\lambda) \omega(k)}, \quad \forall \mu>0 .
\end{aligned}
$$

By using the property $(\gamma)$, for a large $\mu$ we get $\sum_{k=0}^{\infty} e^{-(\mu-\lambda) \omega(k)}<\infty$.

This implies that $\sum_{k=0}^{\infty} r^{k} \hat{u}(k) \cdot \hat{\theta}(-k)$ is convergent at $r=1$. By applying Abel's limit theorem [1, p.41] we get

$$
\lim _{r \rightarrow 1^{-}} \sum_{k=0}^{\infty} r^{k} \hat{u}(k) \cdot \hat{\theta}(-k)=\sum_{k=0}^{\infty} \hat{u}(k) \cdot \hat{\theta}(-k) .
$$

If we put $u_{+}(\theta)=\sum_{k=0}^{\infty} \hat{u}(k) \cdot \hat{\theta}(-k)$ then $u_{+}: \mathcal{P}_{\omega} \rightarrow \mathbf{C}$ is clearly a linear mapping. To show that $u_{+}$is continuous, let $\theta_{m} \rightarrow 0$ as $m \rightarrow \infty$ in $\mathcal{P}_{\omega}$. Now

$$
\begin{aligned}
\left|u_{+}\left(\theta_{m}\right)\right| & =\left|\sum_{k=0}^{\infty} \hat{u}(k) \cdot \hat{\theta}_{m}(-k)\right| \\
& \leq C\left\|\theta_{m}\right\|_{\mu} \sum_{k=0}^{\infty} e^{-(\mu-\lambda) \omega(k)} \quad \text { for a sufficiently large } \mu>0, \\
& \leq C_{1}\left\|\theta_{m}\right\|_{\mu} \text { for some } 0<C_{1}<\infty .
\end{aligned}
$$


Since $\theta_{m} \rightarrow 0$ as $m \rightarrow \infty, u_{+}\left(\theta_{m}\right) \rightarrow 0$ as $m \rightarrow \infty$. Therefore $u_{+} \in \mathcal{P}_{\omega}^{\prime}$.

Now $\hat{u}_{+}(j)=\sum_{k=0}^{\infty} \hat{u}(k) \cdot \hat{e}_{-j}(-k)=\left\{\begin{array}{lll}\hat{u}(j) & \text { if } & j \in \mathbf{N}_{0} \\ 0 & \text { if } & j \notin \mathbf{N}_{0} .\end{array}\right.$

Similarly we can show that if $F_{-}(z)=\sum_{k \in \mathbf{N}} \hat{u}(k) z^{k}$ then $F_{-} \in H(U)$ and if $u_{-}=$ $\lim _{r \rightarrow 1^{-}} F_{-}\left(r e^{i t}\right)$ then $u_{-} \in \mathcal{P}_{\omega}$ with $\hat{u}_{-}(k)=\left\{\begin{array}{lll}\hat{u}(-k) & \text { if } & k \in \mathbf{N} \\ 0 & \text { if } \quad k \notin \mathbf{N}\end{array}\right.$

Hence we get $\left(u_{+}+\check{u}_{-} \hat{)}(k)=\hat{u}_{+}(k)+\hat{u}_{-}(-k)=\hat{u}(k)\right.$. By using Theorem 2.3, the lemma follows.

Definition 5.3. $y \in \mathcal{B}_{A}$ is said to be regular if there exists a representative $f_{n} / d_{n}$ of $y$ such that $d_{n}(z)=\sum_{k=0}^{\infty} \hat{\delta}_{n}(k) z^{k}$ for some $\left(\delta_{n}\right) \in \Delta$.

Let a regular Boehmian $\left[f_{n} / d_{n}\right] \in \mathcal{B}_{A}$ be given. By Lemma 5.1, we have $\lim _{r \rightarrow 1^{-}} f_{n}\left(r e^{i t}\right)$ exists in $\mathcal{P}_{\omega}$, and we denote the limit as $\theta_{n}\left(e^{i t}\right), \forall n \in \mathbf{N}$. First we show that $\theta_{n} / \delta_{n}$ is a quotient. We have the following equalities :

$$
f_{n}(z)=\sum_{k=0}^{\infty} \hat{\theta}_{n}(k) z^{k}, \forall z \in U
$$

and hence (by using the fact that $f_{n} / d_{n}$ is a quotient) we get

$$
\hat{\theta}_{n}(k) \cdot \hat{\delta}_{m}(k)=\hat{\theta}_{m}(k) \cdot \hat{\delta}_{n}(k), \forall m, n \in \mathbf{N}, k \in \mathbf{N}_{0} .
$$

This implies that $\left(\theta_{n} * \delta_{m} \hat{)}(k)=\left(\theta_{m} * \delta_{n}\right) \hat{(k)}, \forall m, n \in \mathbf{N}, \forall k \in \mathbf{N}_{0}\right.$. Using $\hat{\theta}_{n}(-k)=0, \forall k \in \mathbf{N}, \forall n \in \mathbf{N}$, with the above equalities we get

$$
\left(\theta_{n} * \delta_{m}\right) \hat{(}(k)=\left(\theta_{m} * \delta_{n}\right) \hat{)}(k), \forall m, n \in \mathbf{N}, \forall k \in \mathbf{Z}
$$

Theorem 2.1 implies that

$$
\theta_{n} * \delta_{m}=\theta_{m} * \delta_{n}, \forall m, n \in \mathbf{N} \text {. }
$$

Hence $\left[\theta_{n} / \delta_{n}\right] \in \mathcal{B}_{\omega}$.

Next we show that if $g_{n} / e_{n} \sim f_{n} / d_{n}$ and $e_{n}(z)=\sum_{k=0}^{\infty} \hat{\epsilon}_{n}(k) z^{k}$ for some $\left(\epsilon_{n}\right) \in \Delta$, then $\theta_{n} / \delta_{n} \sim \eta_{n} / \epsilon_{n}$ where $\eta_{n}\left(e^{i t}\right)=\lim _{r \rightarrow 1^{-}} g_{n}\left(r e^{i t}\right) . f_{n} \star e_{m}=g_{m} \star d_{n}, \forall m, n \in \mathbf{N}$ implies that $\hat{\theta}_{n}(k) \cdot \hat{\epsilon}_{m}(k)=\hat{\eta}_{m}(k) \cdot \hat{\delta}_{n}(k), \forall m, n \in \mathbf{N}, \forall k \in \mathbf{N}_{0}$.

Thus we have $\left(\theta_{n} * \epsilon_{m}\right) \hat{(}(k)=\left(\eta_{m} * \delta_{n}\right) \hat{(}(k), \forall m, n \in \mathbf{N}, \forall k \in \mathbf{N}_{0}$.

Since $\hat{\theta}_{n}(-k)=\hat{\eta}_{n}(-k)=0, \forall n, k \in \mathbf{N}$ we get that

$$
\left(\theta_{n} * \epsilon_{m}\right) \hat{(}(k)=\left(\eta_{m} * \delta_{n} \hat{)}(k), \forall m, n \in \mathbf{N}, \forall k \in \mathbf{Z} .\right.
$$

Hence we have $\theta_{n} * \epsilon_{m}=\eta_{m} * \delta_{n}, \forall m, n \in \mathbf{N}$. Now we can define the following:

Definition 5.4. Boundary value of a regular Boehmian $\left[f_{n} / d_{n}\right] \in \mathcal{B}_{A}$ is defined by $\left[\theta_{n} / \delta_{n}\right]$ where $\theta_{n}=\lim _{r \rightarrow 1^{-}} f_{n}(z)$, and $\left(\delta_{n}\right) \in \Delta$ corresponds to $\left(d_{n}\right)$.

The following theorem is the main result of this paper.

TheOREM 5.5. Any given $x \in \mathcal{B}_{\omega}$ can be written as $x_{+}+\check{x}_{-}$where $x_{+}$and $x_{-}$ are boundary values of certain analytic Boehmians. 
Proof. Let $x=\left[\theta_{n} / \delta_{n}\right] \in \mathcal{B}_{\omega}$ be given. Define for each $n \in \mathbf{N}$,

$$
\begin{aligned}
& f_{n}(z)=\sum_{k=0}^{\infty} \hat{\theta}_{n}(k) z^{k}, z \in U \\
& d_{n}(z)=\sum_{k=0}^{\infty} \hat{\delta}_{n}(k) z^{k}, \quad z \in U .
\end{aligned}
$$

By Lemma 5.1, we get that $f_{n}, d_{n} \in G$ for each $n \in \mathbf{N}$.

Now we show that $\left(d_{n}\right)$ is a delta sequence in the context of analytic Boehmians. Using mean-value theorem, we get that

$$
\begin{aligned}
\left|\hat{\delta}_{n}(k)-1\right| & \leq \frac{1}{2 \pi} \int_{-\pi}^{\pi}\left|e^{-i k t}-1\right|\left|\delta_{n}(t)\right| d t \\
& \left.\leq \frac{1}{2 \pi} \int_{-\pi}^{\pi}\left|k t^{\prime}\right|\left|\delta_{n}(t)\right| d t \quad \text { (for some } t^{\prime} \text { between } 0 \text { and } t\right) \\
& \leq s\left(\delta_{n}\right)|k| \frac{1}{2 \pi} \int_{-\pi}^{\pi}\left|\delta_{n}(t)\right| d t \\
& \left.\leq M s\left(\delta_{n}\right)|k|, \quad \text { (by the property (ii) of }\left(\delta_{n}\right) .\right)
\end{aligned}
$$

Again by using the property (ii) of $\left(\delta_{n}\right)$ we get $\left|\hat{\delta}_{n}(k)\right| \leq M \forall n \in \mathbf{N}, k \in \mathbf{Z}$. Therefore $\left(d_{n}\right)$ is a delta sequence.

$\theta_{n} / \delta_{n}$ is a quotient implies that $\theta_{n} * \delta_{m}=\theta_{m} * \delta_{n}, \forall m, n \in \mathbf{N}$.

Therefore we get that $\left(\theta_{n} * \delta_{m}\right) \hat{)}(k)=\left(\theta_{m} * \delta_{n}\right) \hat{)}(k), \forall m, n \in \mathbf{N}, k \in \mathbf{N}_{0}$.

i.e., $\hat{\theta}_{n}(k) \cdot \hat{\delta}_{m}(k)=\hat{\theta}_{m}(k) \cdot \hat{\delta}_{n}(k), \forall m, n \in \mathbf{N}, k \in \mathbf{N}_{0}$.

Now $\left(f_{n} \star d_{m}\right)(z)=\sum_{k=0}^{\infty} \hat{\theta}_{n}(k) \hat{\delta}_{m}(k) z^{k}=\sum_{k=0}^{\infty} \hat{\theta}_{m}(k) \hat{\delta}_{n}(k) z^{k}=\left(f_{m} \star d_{n}\right)(z)$. Hence $\left[f_{n} / d_{n}\right] \in \mathcal{B}_{A}$.

Using Lemma 5.1, we get that $\lim _{r \rightarrow 1^{-}} f_{n}\left(r e^{i t}\right)$ exists for each $n \in \mathbf{N}$. We denote this limit as $\theta_{n}^{+}$. Now we show that $\theta_{n}^{+} / \delta_{n}$ is a quotient. For all $k \in \mathbf{N}_{0}$, $\left(\theta_{n}^{+} * \delta_{m}\right) \hat{)}(k)=\hat{\theta}_{n}(k) \cdot \hat{\delta}_{m}(k)=\hat{\theta}_{m}(k) \cdot \hat{\delta}_{n}(k)=\left(\theta_{m}^{+} * \delta_{n}\right) \hat{(}(k)$.

If $k \notin \mathbf{N}_{0}$ then $\hat{\theta}_{n}^{+}(k)=\hat{\theta}_{m}^{+}(k)=0$ which implies $\left(\theta_{n}^{+} * \delta_{m}\right) \hat{(k)}$ $=\left(\theta_{n}^{+} * \delta_{m}\right) \hat{(}(k)$. Hence by Theorem 2.1, it follows that

$$
\theta_{n}^{+} * \delta_{m}=\theta_{m}^{+} * \delta_{n}, \quad \forall m, n \in \mathbf{N}
$$

Therefore $x_{+}=\left[\theta_{n}^{+} / \delta_{n}\right] \in \mathcal{B}_{\omega}$.

By definition $x_{+}$is the boundary value of $\left[f_{n} / d_{n}\right]$. Further we have $\hat{x}_{+}(k)=\left\{\begin{array}{lll}\hat{x}(k) & \text { if } & k \in \mathbf{N}_{0} \\ 0 & \text { if } & k \notin \mathbf{N}_{0}\end{array}\right.$.

Similarly, we can show that if we define

$$
g_{n}(z)=\sum_{k \in \mathbf{N}} \hat{\theta}_{n}(-k) z^{k}, \text { and } e_{n}(z)=\sum_{k \in \mathbf{N}} \hat{\delta}_{n}(-k) z^{k},
$$

then $\left[g_{n} / e_{n}\right] \in \mathcal{B}_{\omega}$ and if $\theta_{n}^{-}=\lim _{r \rightarrow 1^{-}} g_{n}\left(r e^{i t}\right)$ then $x_{-}=\left[\theta_{n}^{-} / \check{\delta}_{n}\right] \in \mathcal{B}_{\omega}$ and hence $x_{-}$ is the boundary value of $\left[g_{n} / e_{n}\right]$.

We also note that $\hat{x}_{-}(k)=\left\{\begin{array}{lll}\hat{x}(-k) & \text { if } & k \in \mathbf{N} \\ 0 & \text { if } & k \notin \mathbf{N} .\end{array}\right.$ 
Finally, we have $\left(x_{+}+\check{x}_{-}\right) \hat{(}(k)=\hat{x}(k), \quad \forall k \in \mathbf{Z}$. Hence by Theorem 3.11 our result follows.

By the following theorem we establish the consistency of this theory with the boundary value theory on $\mathcal{P}_{\omega}^{\prime}$.

TheOREM 5.6. Let $u \in \mathcal{P}_{\omega}^{\prime}$ and $x=\left[\left(u * \epsilon_{n}\right) / \epsilon_{n}\right]$ for some $\left(\epsilon_{n}\right) \in \Delta$.

If $u=u_{+}+\check{u}_{-}$where $u_{+}, u_{-}$are boundary values of some $F_{+}, F_{-} \in H(U)$ respectively, and $x=x_{+}+\check{x}_{-}$where $x_{+}, x_{-}$are boundary values of some $y_{+}, y_{-} \in \mathcal{B}_{A}$ respectively in accordance with Theorem 5.5. Then $x_{+}=\left[\left(u_{+} * \epsilon_{n}\right) / \epsilon_{n}\right], x_{-}=\left[\left(u_{-} * \epsilon_{n}\right) / \epsilon_{n}\right]$ for some $\left(\epsilon_{n}\right) \in \Delta$ and $y_{+}=\left[\left(F_{+} \star d_{n}\right) / d_{n}\right], y_{-}=\left[\left(F_{-} \star d_{n}\right) / d_{n}\right]$ for some delta sequence $\left(d_{n}\right)$. where

Proof. From the proof of Theorem 5.5, we have, $y_{+}=\left[f_{n} / d_{n}\right], y_{-}=\left[g_{n} / e_{n}\right]$

$$
f_{n}(z)=\sum_{k=0}^{\infty}\left(u * \epsilon_{n} \hat{)}(k) z^{k}=\sum_{k=0}^{\infty} \hat{u}(k) \cdot \hat{\epsilon}_{n}(k) z^{k}=\sum_{k=0}^{\infty} \hat{u}(k) z^{k} \star \sum_{k=0}^{\infty} \hat{\epsilon}_{n}(k)\right.
$$

and $d_{n}(z)=\sum_{k=0}^{\infty} \hat{\epsilon}_{n}(k) z^{k}$ and hence $f_{n}(z)=\left(F_{+} \star d_{n}\right)(z)$.

Similarly we get

$$
g_{n}(z)=\sum_{k=1}^{\infty}\left(u * \epsilon_{n} \hat{)}(-k) z^{k}=\sum_{k=1}^{\infty} \hat{u}(-k) \cdot \hat{\epsilon}_{n}(-k) z^{k}=\sum_{k=1}^{\infty} \hat{u}(-k) z^{k} \star \sum_{k=1}^{\infty} \hat{\epsilon}_{n}(-k),\right.
$$

where $e_{n}(z)=\sum_{k=1}^{\infty} \hat{\epsilon}_{n}(-k) z^{k}$ and hence $g_{n}(z)=\left(F_{-} \star e_{n}\right)$.

Therefore we get $y_{+}=\left[F_{+} \star d_{n} / d_{n}\right]$ and $y_{-}=\left[F_{-} \star e_{n} / e_{n}\right]$. This completes the proof of the theorem.

EXAMPLE 5.7. Let $\left(\sigma_{n}\right)$ be a sequence of continuous functions on $\mathbf{T}$ satisfying $\hat{\sigma}\left(2^{k}\right)=0, \forall k \geq n$ and properties (i), (ii), (iii) of delta sequence. For the existstence of $\left(\sigma_{n}\right)$ we refer the reader to [5]. Put $\phi_{n}=\sigma_{n} * \epsilon_{n}$ where $\left(\epsilon_{n}\right)$ is arbitrary delta sequence. Then $F=\left[\left(\sum_{k=1}^{\infty} e^{2^{k} \omega\left(2^{k}\right)} \hat{\phi}_{n}\left(2^{k}\right) e^{i 2^{k} t}\right) / \phi_{n}\right] \in \mathcal{B}_{\omega}$ and $\hat{F}\left(2^{k}\right)=e^{k \omega(k)}, k \in$ N. Hence $F \notin \mathcal{B}_{\omega} \backslash \mathcal{P}_{\omega}^{\prime}$, by Theorem 2.4.

The above example shows that the space of periodic Beurling Boehmians is properly larger than the space of periodic Beurling distribution. If we change the definition of $\Delta$ as the collection of all sequences $\left(\epsilon_{n}\right)$ satisfying

- There exists $M>0$ such that $\left|\hat{\epsilon}_{n}(k)\right| \leq M, \forall k \in \mathbf{Z}$,

- There exists a sequence $\left(\alpha_{n}\right)$ converging to zero such that $\left|\hat{\epsilon}_{n}(k)-1\right| \leq$ $\alpha_{n}|k|, \forall k \in \mathbf{Z}$,

then we get that all analytic Boehmians are regular.

Acknowledgement. The authors would like to thank Prof. D. Nemzer for providing us with the Example 5.7. They also thank the referees for their valuable suggestions towards the improvement of the content of this paper. 


\section{REFERENCES}

[1] L. Ahlfors, Complex Analysis, Mc Graw-Hill, Singapore, (1979).

[2] M.G. Collier and J.A. Kelingos, Periodic Beurling Distributions, Acta Math. Hung., 42:3-4 (1983), pp. 261-278.

[3] V. Karunakaran, Bieberbach Functions and Periodic Distributions, Internat. J. Math. \& Math. Sci., 12:1 (1989), pp. 69-75.

[4] D. Nemzer, Periodic Boehmians, Internat. J. Math. and Math. Sci., 12:4 (1989) pp. 685-692.

[5] D. Nemzer, Periodic Boehmians II, Bull. Austral. Math. Soc., 44 (1991), pp. 271-278.

[6] P. Mikusiński, Convergence of Boehmians, Japan J. Math., 9 (1983), pp. 159-179.

[7] W.Rudin, Functional Analysis, Mc Graw Hill, New York, (1973). 
